\title{
POBLACIONES NATURALES Y CULTIVO DE CIANOBACTERIAS MARINAS DIAZOTROFICAS DEL LITORAL DEL PACIFICO PERUANO
}

\author{
HAYDEE MONTOYA T. 12 \\ MARIO BENAVENTE P. ${ }^{2}$ \\ CARLA AGUILAR S, \\ Labceatorio de Botinica, Universidad Ricardo Palma, Facultad de Ciencias Biologicas."
Laboratorio de Simbensis Vegetal. Universidad Nacional Mayor de San Marcos. Museo de Historia Nasural "2
Cencro de Investigacion para el Desarrobo de la Acuicultura. AQUAC?
}

\section{RESUMEN}

La distribución de las cianobacterias heterocistadas en las orillas rocosas esta relacionada principalmente con el eiclo del carbono y nitrógeno del ecosistema marino. El muestreo de comunidades algales como matas cianobacteriales (epiliticas y epifiticas) en zonas intermareales e supramareales fueron realizados irregularmente a lo largo del litoral del Pacifico entre playa EI Dorado $\left(9^{\circ} 10^{\circ}\right.$ LS) y Puerto Ilo $\left(17^{\circ} 39^{\circ}\right.$ LS). El aislamiento y cultivo de las cianobacterias masinas diazotróficas Calorhrix cnastacea Thuret y $C$. scopulonum Agardh (Rivulariaceae) se tealizó usando el medio $\mathrm{f} / 2$. La variabilidad morfológica entre las diferentes cepas de $C$. crustacea con diversos estados vegetativos (ecomorfos) y reproductivos evidenciaron su alta plasticidad fenotipicat. La sucesión estacional de la cepa de Chala de $C$. crastacéa en laboratorio evidenció su mejor crecimiento entre mediados de invierno y fines de primavera de 1992 (agosto - noviembre) con un rango de temperatura entre 17 y $27^{\circ} \mathrm{C}$. Interacciones de $\mathrm{C}$. crustacea con especies asociadas como C. scopulorim Agardh y Lygbya lutea (Ag,) Gom. permite comprender la dinámica poblacional de las comunidades algales a lo largo del litotal marino. Las especies estudiadas son nuevos registros para la flora marina peruana.

Palabras Claves: cianobacteria, camanidad epilitica, cultivo, heterocisto,

\section{SUMMARY}

Natural Populations and Culture of Marine Diazotrophic Cyanobactena from Peruvian Pacific Litoral.

Distribution of diazotrophic cyanobacteria with heterocystous forms characteristic of rocky shores is related mainly with the cycling of carbon and nitrogen in the marine ecosystem. Sampling of cyanobacterial mat communities (epilthic, epiphytic). at the intertidal and supratidai zones were carried out irregularly along the Pacific ittorai between El Dorado beach $\left(9^{\circ} 10\right.$ S) and Ho Harbor $\left(17^{\circ} 39\right.$ S). Isolation and culture of the marine cyancbacteria Rivulariaceae: Calothrix crustacea Thuret and $C$. scopuionm Agaron were using $1 / 2$ medium. Morphological variability between the different strains of C. crustaces with several vegetative (ecomorphs) and reproductive stages evidenced its high phenotypic plasticity. Seasonal succession of C. crustacea strain of Chala beach evidenced the best growth during mid winter and late spring in 1992 (august-november) within a temperature range between 17 and $27^{\circ} \mathrm{C}$ in laboratory. Interactions with associated species such as C. scopulorum and Lyngbya futea (Ag.) Gom. let us begin to understarid the population dynamics of algal communities along the marine littoral. The studied species are new records for Peruvian marine flora.

Key Words: cyanobacteria, epilathic community, culture, heterocyst.

*Trabejo de investigación presentado partialmente en el XV Simposio fnteinacional de Algas Marinas realizado en enero de 1995 en la Universidad Austral de Chile, Valdivia, Chile. 


\section{INTRODUCCION}

El ecosistema matino de orilla rocosa contiene frecuentemente una variedad de hábitass que sirven de sustrato para organismos de las diferentes comunidades biológicas. La región intermareal es un ambiente dinámico y turbulento que experimenta fluctuaciones frecuentes. Los patrones de zonación en las orillas rocosas intermareales son particularmente potables. Esta zonaciōn se presenta en todo el litoral marino del mundo y los límiles entre los diferentes organismos son frecuentementc más bien imprecisos y uniformes que permite concluir que hay una relación causal directa entre los límites y el nivel del agua en determinados estados de las mareas (DRING, 1982; LUBCHENCO, 1980; LITTLE \& KITCHING,1996).

Las ciancbacterias (cianofitas, algas azul verdes) tienen una histona marina muy antigua $y$ presentan una considerable diversidad y abundancia en los hábitats marinos. Las matas cianobacteriaies son el principal componente de algunos hábitats inter y submareales, ast como los hipersalinos marinos (GOLUBIC, 1976: VINCENT et at. 1993). Las cianobacterias están notablemente bien adaptadas al ambiente marino y deben resistir amplias variaciones en la composición quimica del agua y la acción casi constante de las mareas. De ésta forma han evolucionado para convertirse en integrantes del ecosistema intermareal donde cada especie algal ocupa una distribución vertical caracteristica y un tipo de microbábitat en Ia orilla rocosi.

Las cianobacterias beterocistadas, fijadoras de nitrógeno (diazotróficas) son lipicas de las orillas rocosas y predominan entre las algas del litoral. La fijación de nitrógeno por algunas de sus especies ha sido demostrada por STEPHENSON \& STEPHENSON (19.49), STEWART (1965) y GALLON \& CHAPLIN (1988) Algunas ventajas selectivas para la dominancia de éste grupo representan una estrategia alternativa de particular Televancia para comprender la ecología cianobacterial (WHITTON, 1992).

El presente trabajo está relacionado

con las cianobacterias marinas heterocistadas Calothrix crustacea Thuret y C. scopulorien Agardh (Rivulariaceac) con la finalidad de describirlas con énfasis en su forma de crecimiento y reproducción de poblaciones naturales $y$ en cultivo unialgales de laboratorio. Además caracterizar la estructura y ocologia de sus comunidades cianobacteriales epiliticas y epiffiticas.

\section{MATERIAL Y METODOS}

Las orillas rocosas son comunes a lo largo del fitoral costero del territorio peruano. Colecciones ifregulares de las comunidades algales en zonas inter $y$ supramareales fueront realizadas entre la playa El Dorado $\left(9^{*} 10^{\circ}\right.$ LS) y Puerto tlo (17 $39^{\prime}$ LS) entre 1983 y 1994

La obtención de secciones de matas cianobacteriales del sustrato, así como porciones de rocas con comanidades algales fueron extrafdas. Registros de temperatura (termómetro de $-10^{\circ} \mathrm{C}$ a $110^{\circ} \mathrm{C}$ ) y salinidad (salinometro A.T.T/C) fueron realizados durante lis colecciones. Para estimular el crecimiento de determinadas especies y mantener las matas algales vivas con su integridad estructural, las muestras fueton transportadas en su propia de mar. El aislamiento $y$ cultivo de las cianobacterias marinas fueton realizadas usando el medio $f / 2$. con salinidad de 35 o/Oo y $\mathrm{pH} 7$ (GUILLARD, 1975). Se realizaron inoculaciones estándar de las poblaciones algales y las técnicas de cultivo fueron aplicadas en medio. líquido y en placas (agar $1.5 \%$ ). Los cultivos fueron mantenidos en condiciones de taboratorio con un rango de temperatura de $17-31^{\circ} \mathrm{C}$ con iluminación natural. La evaluación biométrica en las diferentes cepas fueron realizadas mediante observación microsoópica y la identificación de especies mediante consulta con bibliografia especializada.

\section{RESULTADOS}

\section{AREA DE ESTUDIO}

Las orillas rocosas a lo largo del litoral peruano proporcionan una variedad de microhábitats con una diversidad de 
crganismos como las cianobacterias, Las estaciones de muestreo fueron de sur a norte. en el departamento de Moquegua: Puerto de I!o $\left(17^{\circ} 39^{\circ}\right.$ LS, $71^{\circ} 22^{\circ}$ LO): departamento de Arequipa: playa de Chala $\left(15^{\circ} 51^{\circ}\right.$ LS, $\left(74^{\circ} 15^{\prime}\right.$ LO) ; departamento de lca: Mendieta (14. 01' LS, 76 $16^{\circ}$ LO) y Lagunilla $\left(13^{\circ} 53^{\circ} \mathrm{LS}, 76^{\circ} 18^{\circ} \mathrm{LO}\right)$ en la Bahia de Paracas; departamento de Lima: playa Ancón $\left(11^{\circ} 46^{\circ} \mathrm{LS}, 77^{\circ} 12^{\circ} \mathrm{LO}\right)$ y departamento de Ancash: playa El Dorado $\left(9^{\circ} 10^{\circ}\right.$ LS, $78^{\circ} 32^{\prime}$ LO $)$.

Comanidades epiliticas y epifitas en las zonas inter y supramareal constituidas por matas cianobsacteriales están expuestas a grandes variaciones por el grado de alteraciones (acción de oleaje) y estrés (desecación. efecto termal) que ocurren dentro de áreas relativamente pequeñas. Las cianobacterias usualmente cubren las rocas así como las fisuras y grietas como una capa o película continua 0 como parches asslados de tal forma que las rocas frecuentemente tienen apariencia negruzca por éstas capas algales encrustantes. En baja marea. las zonas ínter y supramareales son separadas del oceano, permaneciendo agua de mar aislada en pozas de marea. En éstos habitats efimeros del litoral rocoso donde el nivel de agua disminuye gradualmente, las comunidades de cianobacterias pucden llegar ccasionalmente a secarse. Fig. 1 y 2.

\section{DESCRIPCION DE ESPECIES}

Las descripciones de las especies diazotróficas incluyen datos de las poblaciones naturales y de clones aislados en sultivo de laboratorio. La amplia distribución de poblaciones epifiticas de $C$. cnustacea en la mayoria de estaciones muestreadas es reportada como comunidades algales florecientes (Figs. 58).

\section{Calothrix crastacea Thuret}

Las cepas de Chala, Do, El Dorado y Antón han sido reconocidas muy estrechamente por sus caracteristicas similares. El talo se presenta fasciculado, como penachos agregados, crustoso, azul verde, verde amarillento o pardo oscuro, de textura gelatinosa o más bien cơriácea cuando se seca.

Filamentos ondulados cortos o largos, de disposición paralela como densos fasciculos o laxamente juntos y entrelazados. Distribución paralelà de los tricomas heterocistados basalmente curvados que proyectan su extremo distal atenuado hacia la superficie de la mata algal. La ramificación falsa unilateral puede ser continuada en sucesivas ramificaciones. La perción basal de filamentos de 10.5 - 21 $\mu \mathrm{m}$ de diámetro con gradual atenuación hacia el ápice con diámetro de $7.5-9.6 \mathrm{um}$. Filamentos anchos hasta $27 \mu \mathrm{m}$ de diámetro, con divisiones oblicuas que originan hormogonios. Los filamentos con tricomas espiralados pueden dividirse en varios segmentos formando agregados densos de hormogonios que posteriormente se liberan. La élula del extremo del hormogonio es diferenciada eventualmente en un heterocisto basal. Los hormogonios fueron tambièn diferenciados de la parte terminal de los tricomas y su liberación involucró la formación de célula necridial o đisco biónncavo. Células discoidales constrictas o ligeramente constrictas. Diámetro celular en la base de los tricomas de 6.6 a 14.6 um. células intermedias de 4 a $9 \mu \mathrm{m}$ de diámetro y 1.3 a $4 \mu \mathrm{m}$ de longitud. Célula apical redondeada, hemisférica, cónica o algo aguda, azul verde o pálida, de 5.3 a $14.6 \mu \mathrm{m}$ de diámetro y 3.3 a $8.6 \mu \mathrm{m}$ de longitud. Estuche gelatinoso, estratificado paralelamente, incoloro, pardo oscuro o pardo amarillento, de 0.9 a $4 \mu \mathrm{mt}$ de espesot: Generalmente se presenta un heterocisto basal, hemisfético, verde amarillento o amarillo pálido. Hererocisto intercalar ovoide, aislado o por pares, de 5.3 a $14.6 \mu \mathrm{m}$ de diámetro y 3.3 a $8.6 \mu \mathrm{m}$ de longitud.

Varios estados de desarnollo del filamento con formas morfológicamente más complejas se presentaron en cultivos de C. crustacea formando talos densos azul verdes similares a ahmohadillados con rextura aterciopclada. También la formación de asa aislada en tricomas o vuelus muy pronunciadas, con ramificación inicial unilateral o con ramas en pares fueton frecuentes. Las ramificaciones falsas unilaterales consecutivas proporcjonan 
densos penachos de tricomas. Algunas veces desarrollan ramas a nivel de los heterocistos. En cultivos liquidos forman filamentos agregados similares a motas o protuberancias verdes. Tricomas espiralados rodeados por un estuche definido. Filamentos gruesos de expansión radial, con células adultas pálidas. vacuoladas (cultivo de 8 meses). Filamentos largos ateruados con secciones ensanchadas. por la distensión del estuche con agregados de tricomas creciendo curvados y atenuadosicultivo de 2 meses). Células basales con notorio estuche estratificado. Hormogonios frecuentes en los extremos apicales de los filamentos, algunos hormogortios libres rodeados de estuche (encapsulados). En algunos casos los hormogonios son limitados por heterocistos terminales. En verano (fines de enero y febrero) los hormocistos (estados latentes) fueron formados por el encistamiento de hormogonios motiles que migraron al separarse del filamento principal. En cultivos de 1.5 meses, los hormocistos germinaron por fisión y protusión de las células de tricomas de ambos extremos de los hormocistos multicelulares dejando el estuche vacio, posteriormente cada filamento joven elongado crece mís allá del estuche grueso del hormocisto (Fig. 8). Agregados de hormogonios curvados liberados. de filamentos hinchados (cultivos de 5 meses) en primavera (mediados de octubre) fueron rodeados por estuche grueso semejando a hormocistos. Similares hormocistos fueron reconocidos en verano (febrero) en cultivo líquido de 9 meses en filamentos con numerosos heterocistos. Cultivos viejos en placa ( 9 meses) casi secos (condición de desecación en laboratorio) exhibieron filamentos espiralados e irregulares con estuche grueso larnelado y células muerias. C. crustacea estuvo asociada con C. scopulonum. Plearocapsa sp. Oscillatoria sp. y diatomeas pennadas en el estado inicial de su aislamiento.

Las cepas relativamente grandes de C. crestacea de las playas de Mendieta y Lagunilla en la Bahía de Paracas formadas por matas cohesivas compuestas de densos filamentos coalescentes $y$ entrelazados firmemente llegan a formar costras laminares pardo oscuras principalmente en pozas de marea en Lagunilla (Figs.9-12). La proliferación de células algales en filamentos en crecimiento mostraron tricomas azul verdosos. Filamentos largos. ligeramente atenuado5, de 17 a $29.3 \mu \mathrm{m}$ de diámetro con notorio estuche estratificado, pardo amarillento o pardo oscuro. Filamentos con ramas falsas unilaterales o ramas pares (Fig. 9). Diámetro celular de 13.3 a $25.3 \mu \mathrm{m}$ y 2 a $5.3 \mu \mathrm{m}$ de longitud. Algunos tricomas maduros que llevan de I a 3 heterocistos basales en diferentes estados de desarrollo e intercalares en sertie hasta 6 . demostraron el incremento en diámetro celular durante el curso del desarrollo sucesivo del heterocisto (Figs.9.11). Formación frecuente de hormogonios heteroctstados (1 - 4) o aheterocistados rodeados por estuche parental estratificado (Fig. 10). Heterocistos de 12 a $22.6 \mu \mathrm{m}$ de diametro y $4-10.6 \mu \mathrm{m}$ longitud. En cultivos liquidos. el crecimiento algal evidenció tricomas azul verdes y espiralados formando hormogonios $y$ estuche lamelado e incoloro (Fig. 12). 

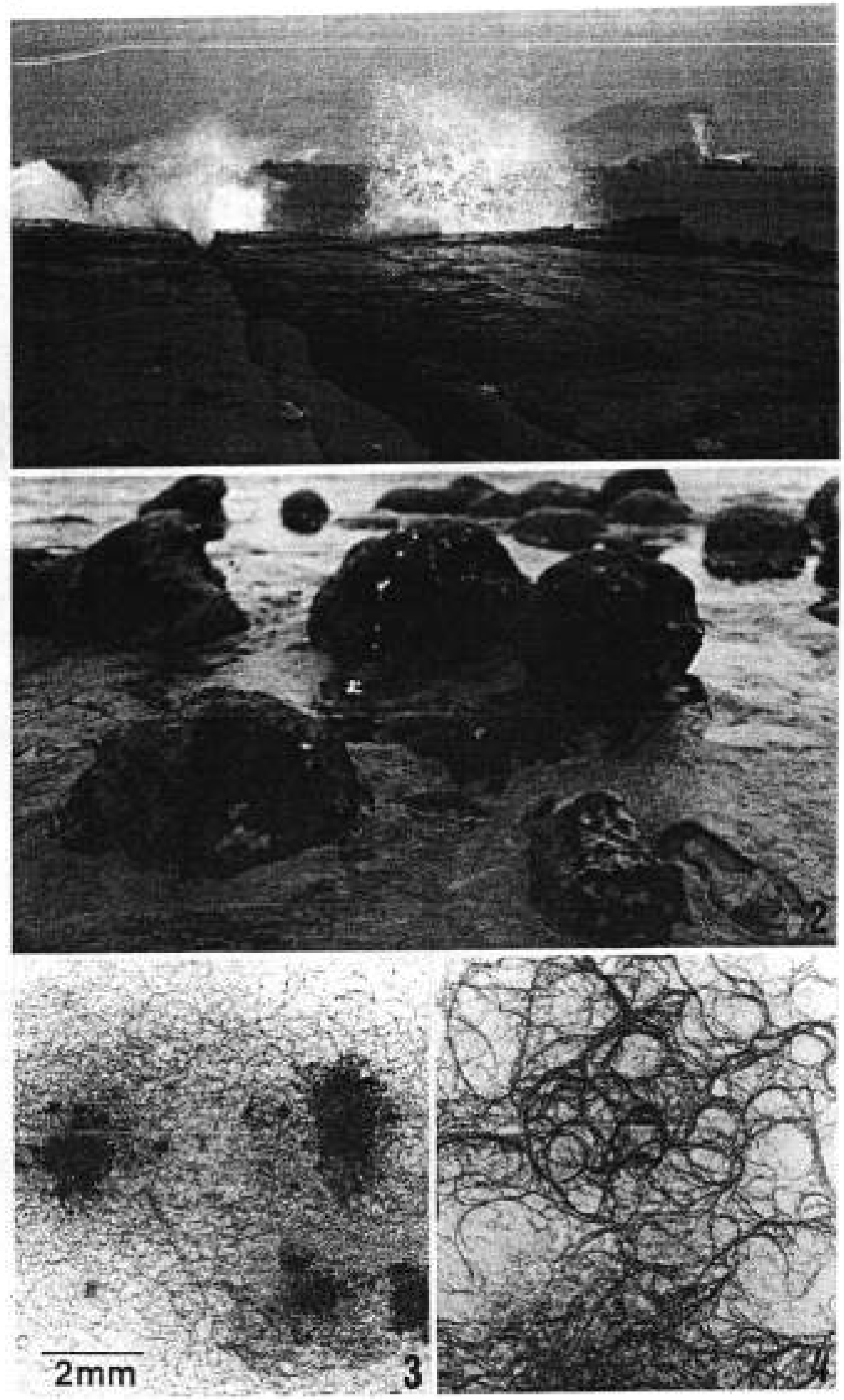

Figura 1 Planicie supramareal en plays Mendieca. Bahía de Paracas con matas cianobocteriales perennes dominada por Calothrix crustacea Thutet penódicamente humedecidas por las olas rompientes en

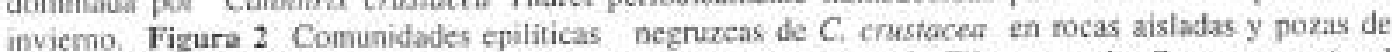
marea de zona supramareal de playa Mendieta, en invierno. Figura 3 Filamentos de $C$. crusiaceso (azul verdest asociabo con los de C. scopuforam Agardh (parduscos) durante el proceso de aislamiento de las cepas cianobacteriales de playa Chala. en coltivos de 3 meses. Figura 4 Filamentos de C crastacea (cepa de Chala) de cultivos unialgales en placa de 5.5 meses. Escala en Figura 3 válida para Figuras 3 4 

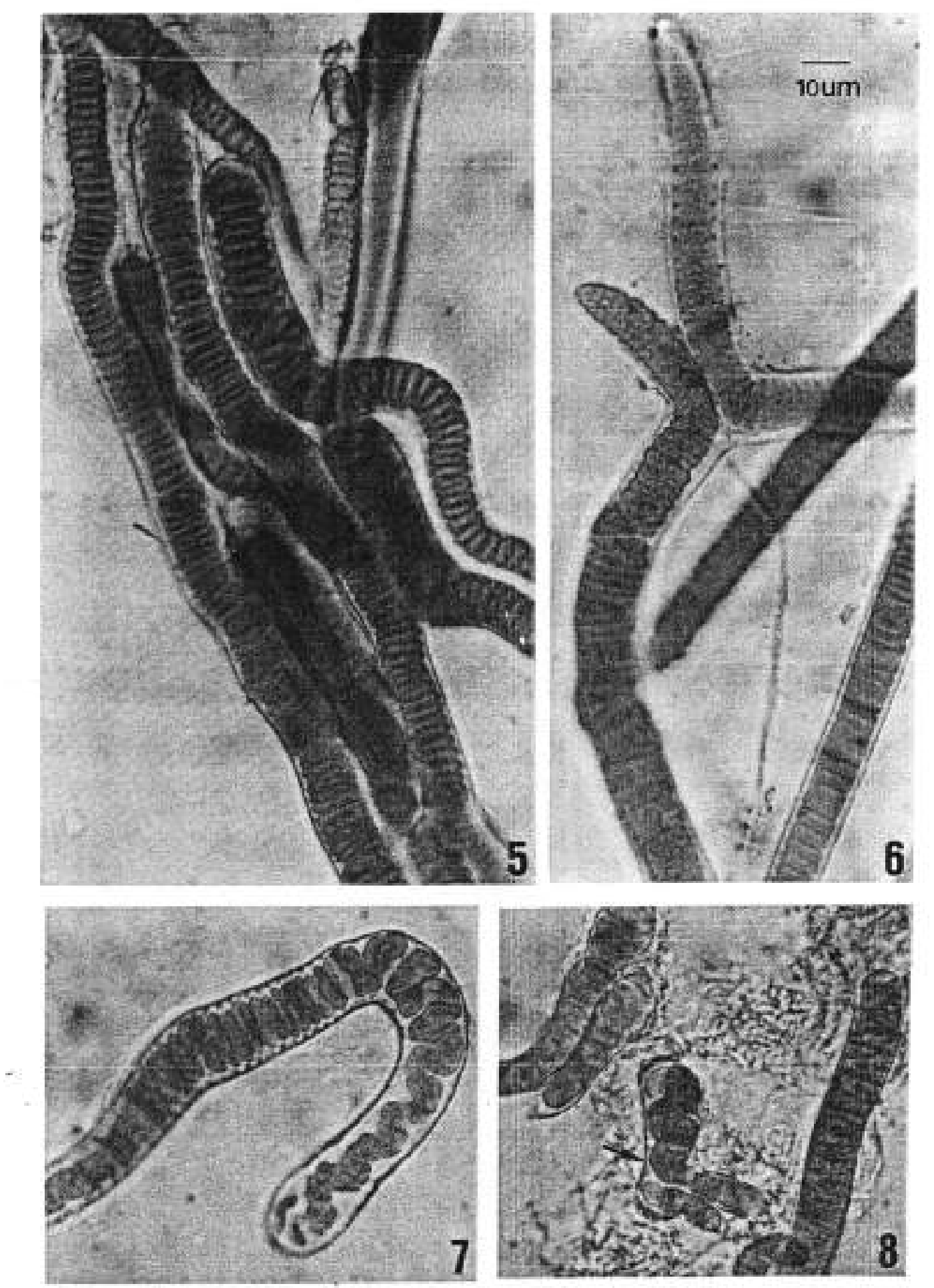

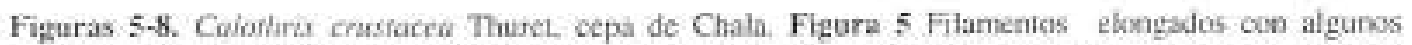
Iricomas atenuabos. Celulas vegetativas discoidales y heterocistos hasales de cultixos ell placa de 5.5 meses. Figura 6 Ramas falsas en pares de filamentos en culivos liquidos de 9 meses. Figura 7 Tricomas espiraladks todeados de un estuche deffinido y grveso de cultivos en placa de 8.5 mesex. Figura 8 Cieminación de hormocistos y liberación de homogonios (ffecha) de cultivos en placa de i.5 meses. Escala en Figura 6 válida para Figuras. 5-8. 

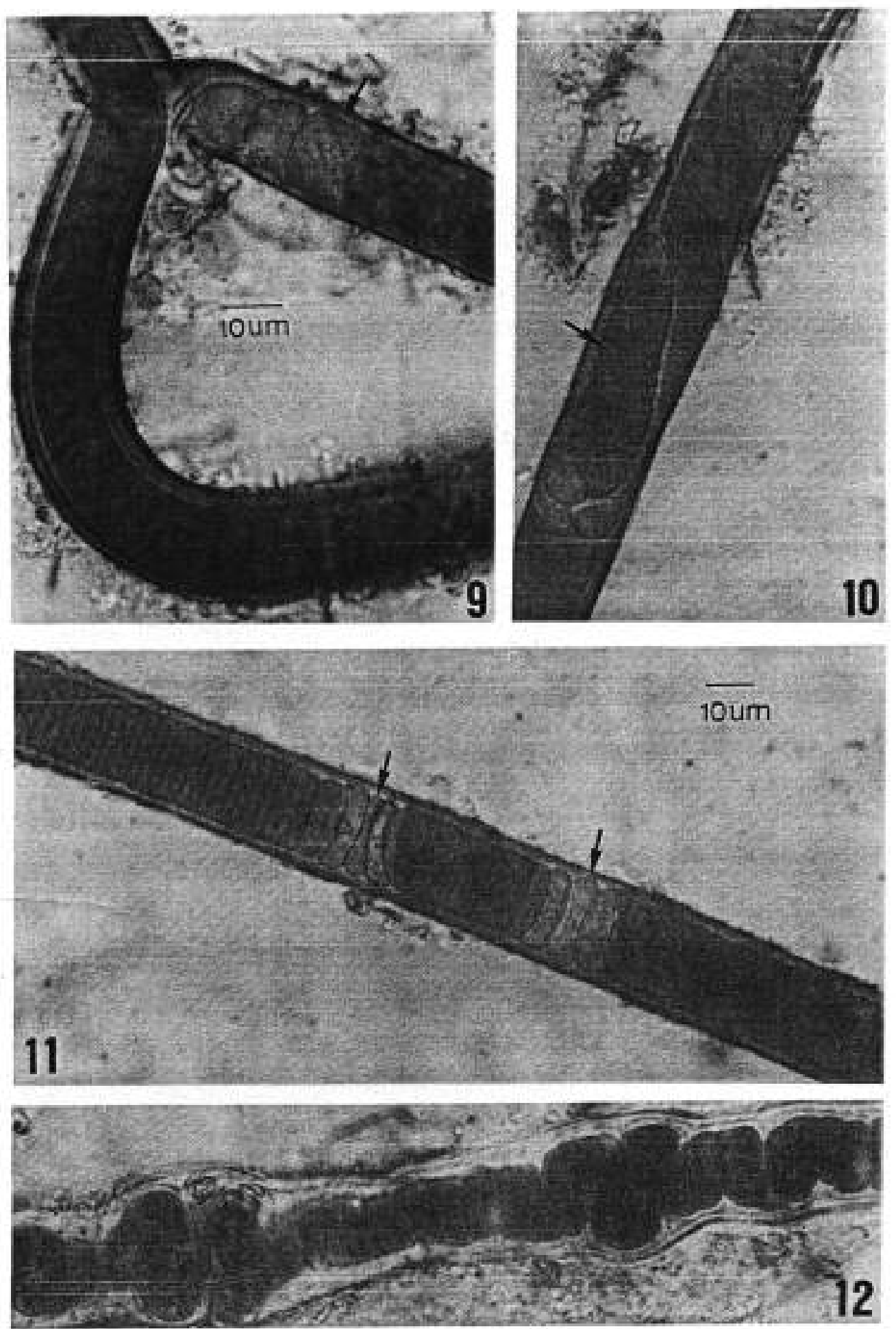

Fizura 9 Caiohrix crustacea Thuret, cepa de Lagunilla. Rama falsa con heterocistos basales seriados (flecha) de poblaciones naturales. Figuras 10-12. C. crusiacea cepa de Mendieta. Figura 10 Formación de bormogonic heterocistado (flecha) dentro del estuche estratificado en poblaciones raturales. Figura. 11 Filamento con frecucnies cadenas de heterociats intercalares (tlechas) en poblacionies naturales. Finura 12 Diferenciación del filamento en porciones hinchadas con tricomas espiralados y subsecuente formación de hormogonios agregsdos rodeados de estuche estratificabo e incoloro en cultivos liquidos de 5.5 meses. Escala en Figura 11 válida para Figuras. 10-12. 

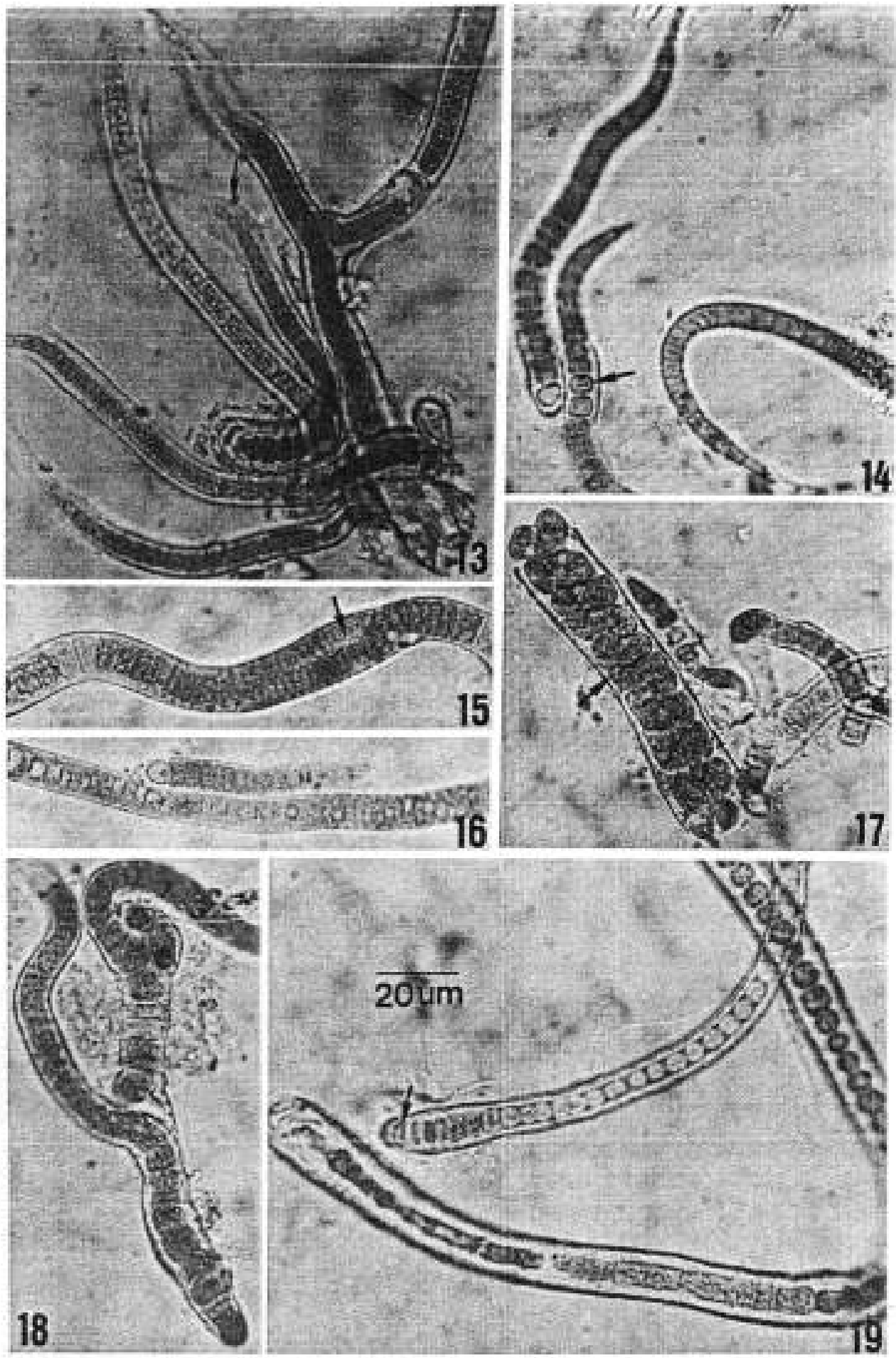

Fìns. 13-19. Cajothirix scopulonim Agardh, cepa de Chala (cultivos en placa), Figura 13 Filamentos elongados atenuados con formación de ramias unilaterales y estuche mucilaginoso definido (flecha) en cultivo de 3.5 meses. Figura 14 Filameatos atenuados con pelo laryo y helerocistos hasal e intercalares (flechas en culivios de 2 meses. Fieura 15 Celulas de filamento heterocistado con divisiones perictinales (ffecha) en cultivos de 2 meses. Fizura 16 Desprenilimiento de fitamento heterocistado dz tale parental (células vacuoladas) en cultivo de 2 meses. Figura 17 Tricomas espiralados (flecha) rodendks de un extuche gruesso y desarrollo de bormogonios en cultives de 2 meses. Figura 18 Filamenlos con ramas unilaterales consecutivas en cultivs de 4 meses. Fizura 19 Filarnentos con esiucbe definido, heterocisno basal nedocido (Recha) y forma celulat variable, en cultivo de 6.5 meses. Escala en Figuna 19 yslido para Figuras 13-19. 


\section{Calorhirix scopulonem Agardh}

Esta especie forma matas almohadilladas parduscas con filamentos elongados, curvados, largos o cortos, gradualmente atcnuados de la base al ápice. El diámetro basal de los filamentos es de 7.3 - 13.3 um. Heterocistos basal e intercalares de 4 a $9.3 \mu \mathrm{m}$ de diámetro y de 3 a $8 \mathrm{\mu m}$ de longitud (Figs, 14-16). Ramificación falsa unilateral algunas veces con la formación de una asa previamente. Tricomas espiralados dentro de un estuche firme. de 12 a $17.3 \mu \mathrm{m}$ de diámetro (Fig. 17). Los tricomas escasamente atenuados dentro de un estuche pardo amarillento 0 pardo verdusco, estratificado y grueso. de hasta $4.6 \mathrm{um}$. Células basales e inlermedias discoidales de 3 a $10.6 \mu \mathrm{m}$ de diámetro y de 1 a $6.6 \mu \mathrm{m}$ de longitud. Células apicales alargadas, cónicas y vacuoladas, alcanzando de 2 a 4 um de diámetro y 1.5 $4.6 \mu \mathrm{m}$ de longitud. (Figs. 13-19).

La porción terminal del tricoma puede metamorfodearse en un pelo largo demostrando la típica polaridad de la especic. El pelo presenta algunas células vacuoladas de 1 a $2.6 \mu \mathrm{m}$ de diámetro. rodeadas de un estuche lamelado amplio e incoloro. La fragmentación intercelular del tricoma en pequeñas porciones origina hormogónios heterocistados. El crecimiento y elongación de los hormogónios conduce eventualmente a la diferenciación de los. tricomas de igual diámetro en los cuales los heterocistos son predominantemente intercalares. En cultivos de 6.5 meśes, las células se presentan en forma de barril y con un estuche firme y grueso. (Fig. 19). Estas observaciones morfológicas fueron hechas en cultivo de cepas de $C$. scopuloram de Chala y Lagunilla.

\section{COMUNIDADES DE CIANOBACTERIAS HETEROCISTADAS}

La colonización de las orillas mocosas fue notoriamente conspicua con predominancia de cianofitas beterocistadas con Calothrix crustacea como especie dominante en comunidades inter y supramareales.

La cepa de $C$. cnustacea de playa Mendieta fue muestreada de hábitats heterogéneos en abril 23, 83; junio 12, 88 y julio 17,93 . Esta especie coloniza y florece formando una capa crustacea gruesa y continua, negruzca o pardo oscura sobre cl sustrato rocoso en una planicie supramareal de aproximadamente $5 \mathrm{~m}$ de altura de la orilla. Densas matas de $C$ crustacea dominan el área cercana al borde de la planicie y se mantienen periódica y constantemente húmedas durante las mareas altas, Este nivel no es continuamente alcanzando por la marea, de tal forma que C crustacea y especies asociadas. capaces de vivir por encima del nivel más alto de la marea, algunas veces quedan completamente sumergidas por e] agua de mar. La comunidad dominante de C. crustacea mantiene su matrix algal perene, húmeda y fria por las olas rompientes. La salpicadura de las olas también cleva el nivel del agua por encima de la altura mareal estimada. Por consiguiente, el tiempo de emersión de $C$. crustacea es reducido en la planicic supramareal de playa Mendieta. Las poblaciones de ésta especie disminuyen conforme la distancia a la orilla aumenta. Además, piedras y rocas aisiadas que permanecen en la superficie de la planicie sirven de substrato a comunidades encrustantes de $C$ crustacea. Las salpicaduras también originan pozas de mareas temporales colonizadas por películas $\delta$ capas mucilaginosas de $C$. crusfacea. Aunque ésta especie proporciona la estructura básica a la comunidad algal, especies asociadas como Pleurocapsa sp. y diatomeas pcrnadas fueron también componentes importantes, En las pozas de marea C. cnustacea se presentó distribuida en parches dentro de la matrix algal pardo amarillenta o pardo verdosa con burbujas de oxígeno atrapadas y una capa azul verde en el interior de lat matrix. Cuando la marea baja $C$. cnustacea forma comunidades expuestas aćreas $y$ subaéreas. Por consiguiente, en Mendieta el principal componente estructural de la mata algal fue C. crusracea dominante localmente y bien adaptada a resistir la desecación a la cual se encuentra constantemente expuesta. Además, ésta extensa comunidad de $C$. crustacea es raria en otros lugares a lo largo de nuestro litoral (Figs. 1.2). 
La cepa de $C$ crustacea de la playa de Lagunilla colectada en julio 17, 93 y cetubre 22, 94 forn6 matas membranosas lameladas, verde oscura. verde pardusca ó amarillo parduscus, asociada con Lyngbya aestuarit y Pleurocapsa sp. en pozas temporales supramareales. Las comunidades epilíticas sumergidas llegan a sccarse y los talos crustáceos y coriáceos formados se quiebran durante los periodos de desecación cubriéndose con sal que los hace casi irreconocibles siendo transportados ocasionalmente por el viento. La gradual desecación de las pozas de marea origina un incremento en salinidad descle 35 a 85 oloo $(\mathrm{NaCl})$ quedando los talos expuestos a estrés salino. A fines de octubre; ta remperatura del agaa en las pozas fue de $31^{\circ} \mathrm{C}$ a las 2 p.m. mientras que el agua de mar estuvo a $17^{\circ} \mathrm{C}$ simultaineamente.

La cepa de $C$. cristacea colectada en la playa de Chala en octubre 7,91 y mayo 1. 92, formo talo saxicola, membranoso y crustacen, azul verde oscuro o pardo negruzco colonicando las zonas inter $y$ supramareales. En focas de rompientes se le observó epifila sobre el talo de Pleuracapsa sp. y epilinica formande costra oscura o verde negruzca colonizanio una plataforma de concreto o rompeolas. $C$. crustacea en zonas intermareales coexiste con las macroalgas Lessonia nigrescens (Phacophyta) y Ulwa costata (Chlorophyta).

La cepa de $C$, crustacea de la playa El Dorado fue colectada en octubre 29, 92, la de playa de Ancón en abril 17,92 y la del puerto de llo en noviembre 1, 91,ellas formaron costras saxicolas verdes oscuras $Q$ parduscas en zonas inter y supramareal det litoral rocoso.

La cepa de C. scopulorum de playa Chala fue colectada en octubre ?,91 y la de Lagunilla en julio 17,93 como talos crustáceos o membranosos, pardo oscuros de las zonas fnter y supramareal.

\section{CULTIVO Y SUCESION DE CIANOBACTERIAS}

Las matas algales fueron removidas del substrato rocoso en Chaia e inmediatamente $s e$ realiz $601 \mathrm{a}$ inoculación de tos theomas de $\dot{C}$. crastacea en primavera (octubre 7, 91) en medio liquido. El primet crecimiento algal estuvo dado por la cianobacteria filamentosa y delgada Oscillatoria sp, quien floreció durante 4 meses a mediados de primavera y verano (noviembre 91 - marzo 92) con un rango de temperaturit entre $24-31^{\circ} \mathrm{C}$. Esta especic formó una mata laminar cohesiva azul verde que llegó a decaer con el tiempo cuando se transformo en matas blanquecinas por lit muerte de sus tricomas.

La sacesión algal en cultivo con la colonización temporal de Oscillatoria sp. de Chala fue evidente. Esta especie floreció a mediados de primavera y verano y fue capaz solamente de resistir un rango de temperatura limitado $\left(24-31^{\circ} \mathrm{C}\right)$ bajo limitacion de nutrientes (cultivos viejos de 5 meses). Después de 6 meses (fines de abril, 92 ) de la inoculación de $C$. crustacea fue defectado su crecimiento inicial como puntos verdosos sobre las matas blanquecinas que constituyen el substrato algal muerto de Oscillatoria sp. Transferencias de $C$. crustacea realizadas en junio 92 (temperatura $22^{\circ} \mathrm{C}$ ) permitió su aislamiento de las especies asociadas. Debido al primer periodo de adaptación de C cruszacea en medio líquido donde floreció Oscillatoria sp. . su crecimiento en placas (junio 92) fue evidente durante 2 meses (lento crecimiento). Diferentes estados vegetatives y reproduclivos fiseron reconocidos cuando floreció por 6 meses durante invierno y primavera (juniodiciembre 92) dentro de un rango de temperatura de $17.27^{\circ} \mathrm{C}$ observándose algunos de sus tricomas muenos en diciembre. Por consiguiente, el mejor crecimiento de $C$. crustacea fue observado entre agosto a noviembre, $92\left(17-27^{\circ} \mathrm{C}\right)$.

C. scopulorum fue una de las principales especies asociadas de C. crustacea que creció en el primer pliagueo en jutio 92. Esta especie fuc claramente distinguible por su color pardusco comparado con la coloración verde azulada de $C$. crustacea (Fig, 3) Esta coexistencia foe mantenida por algunos meses (4) despuess del cual $C$. scopulorum empezó a decaer a mediados de primavera (noviembre 92).

La inoculación en placa de la cepa $C$ crassacea de otra estación de Chala relativamente cercana a la anterior fuc realizada en oton̂o (mayo 4,92 ), Debido a 
1a variedad de crecimiento de otras algas filamentosas, réplicas fueron tealizadas a fines de otoho (junio 11, 92). El mejor desarrollo correspondió a la cianobacteria filamentosa Livgbya lutea scguida por $C$ crustacea. La demora en la colonización de In placa por $C$. crassacea cvidenció su lento crecimiento comparado con $L$ lutea quien creció y domino por aproximadamente 2.5 meses en invierno (junio-agosto,92) dentro de un rango de temperatura de $17-25^{\circ} \mathrm{Ce}$ iniciándose la decadencia de sus filamentos (setiembre,92) luego de su florecimiento. $C$. crustacea que estuvo latente desde su inoculación empezó a crecer en agosto permaneciendo dominante de setiembre a diciembre $\left(19-27^{\circ} \mathrm{C}\right)$. Fig. 4. Esta especie sobrevivió durante la estaciòn de verano (fines de diciembre 92 - marzo 93). No obstante. en febrem, $93 \mathrm{sc}$ reconocio algunos filamentos muertos y en marzo (27 $-3 I^{\circ} \mathrm{C}$ ) filamentos palidos fueron comunes y los verdosos llegaron a ser raros. Por consiguiente, hubo una definida sucesión algal en cultivo por 9 meses donde Lyngbyo lutea. especie asociada de C. crustacech, alcanzó su máximo crecimiento a inicios y mediados de invierno (junio-agosto 92). Sin embargo, $C$. crustacea consiguio proliferar desule fines de invierno a fines de primavera (setiembre - diciembre 92) después de 3 meses de adaptación.

\section{DISCUSION}

Las cianobacterias son altamente exitosas en diferentes ambienes. generalmente predominan en las comunidades epiliticas marinas. Sin embargo, la excesiva alteración en las costas rocosas previenen su colonización y restringen su desarrollo en orillas rocosas expuestas al oleaje. Las especies residentes están morfológica y fisiológicamente equipadas para enfrentar grandes cambios en las condiciones ambientales. Las especies que no fueron adecuadamente adheridas a las focas pueden set barridas por el surgimiento o leve incremento de la acción de la maren, a veces no detectable. Este hecho puede explicar la baja diversidad de especies en las comunidades epilíticas cianobacteriales comparada con la mayoria de otras poblaciones algales no alteradas sugiriendo que el ambiente recoso es relativamente hostil para muchos taxa (MOORE, 1974). En la zona supramareal. los límites superiores para la mayoría de especies han sido hallados ser mayores en los Jugares más expuestos como en las playas de Mendieta y Lagunilla. En Mendieta, $C$ crustacea es la especie local dominanie considerando que es más bien ma en otras estaciones de muestreo. Las matas de $C$. crustacea en Mendieta constituyen microhabitats importantes dentro de la planicie del litoral y alcanzan su mayor abundancia natural en las zonas supramareales bajo condiciones fluctuanles.

La diversidad morfológica y ecológica de las cianobacterias esta relacionada con la colonización de diferentes hábitats marinos. Las especies estudiadas que crecen adheridas a superficies rocosas son morfológicamente más complejas y están relacionadas con ambientes especializados como las zonas inter y supramareales. Tricomas múltiples y espiralados incluidos por un estuche firme común en cultivos de $C$, crustacea y $C$. scopulorion demostraron un efecto de aglomeración de tricomas que crecicron rípidamente, siendo similares a los reportados para las cianobacterias Dichorhrix, Polythrix y $C_{+}$desentica (RIPPKA et al.. 1979).

Muchas cianofitas filamentosas
experimentan cambios morfológicos
cuando estín sujetas a la desecación y limitación de nutrientes como la disminución de nitrógeno. fosforo y fierro. La frecuencia de heterocistos en un tricoma puede reflejar no solamente los niveles de nitrógeno en el tricoma sino también sus niveles de molibdeno, calcio y fierro. Este último es elemento importante en el funcionamiento de la nitrogenasa KIRKBY \& WHITTON 1976. WHITTON, 1992). Enlre las cepas de cjanobacterias diazotróficas aisladas, la más importanle fjadora de nitrógeno en las playas de Mendieta. Lagunilla y Chala fue C. crustaced quien exhibe considerable diversidad en sa morfología, distribución y frecuencia de heterocistos. Las cepas de Mendieta y Lagunilla desarrollaron heterocistos extras, en posiciones basales e intercalares, aislados o en cadenas halsta 
seis. Por consiguiente. es probable que éstas cepas hallan desarrollado estrategias con respecto a la fijación de nitrógeno $y$ algunas ventajas selectivas para su dominancia como diazótrofos perennes.

Las cepas de Calothrix exhiben varias categorias de un solo carácter que evidencian su variabilidad genética. La extensión de desarrollo de los pelos en tricomas de Calothrix pueden variar considerablemente, Los pelos no están presentes en todos los estados de crecimiento siendo más bien liberados cuando se forman y desartollan los hormogonios independientemente. La deficiencia de nutrientes (fosfato y fierto) y los medios de eultivo de baja salinidad producen el desarrollo de pelos en $C$. viguieri Fremy, sin embargo ésta especie no desamolla pelos en un medio completo (WHITTON et al.. 1973; MAHASHNEH et al., 1990). También, el desarrollo del pelo es reducido o suprimido completamente bajo condiciones subaéreas de campo y de laboratorio (DARLEY 1968). La presencia del pelo también fue observada en cultivos de las cepas de C. scopulorien de Chala y Lagunilla. $\mathrm{La}$ coexistencia de $C$ cristacea y $C$ scopaloram en cultivos en placa evidencio una serie de formas de crecimiento. C. scopulorum caracterizada por sus tricomas atenuados y pelos incoloros en presencia de abastecimiento de nitrógeno combinado, sugiere que la formación del pelo está relacionado con su potencial genético comparado con otras cepas beterocistadas las cuales producen pelos en medios exentos de nitrógeno combinado y pierderi completamente la polaridad del tricoma en medios completos. (SINCLAIR \& WHITTON 1977).

Enure los tipos de células especializadas de las cianobacterins, los hormocistos están compuestos de una hilera corta de células-altamente granuladas y completamente rodeadas por un estuche común condensado como en Westiella lanosa (FOGG et al., 1973). La germinación del hormocisto de Scynonema javanicum (Karz) Bornet ha side reportado por ALLSOPP (1968). Cultivos viejos (placas) de C. crustacea en verano pueden ser comparados con la desecación natural y su adaptación a altas temperaturis de verano $(20) 27-31^{\circ} \mathrm{C}$ puede ser atribuida al desarrollo de estados latentes como los hormocistos.

Las cianobacterias helerocistadas estudiadas adheridas al substrato rocoso tienen estuches gruesos producidos por las células del tricoma que ayudan a la adhesion de la mata algal al substrato. Adentás, la pigmentación del estuche puede ayudar a disminuir la intensa radiación solar que alcanza a las células. Así, en poblaciones naturales las células algales más activas se hallan dentro de la mata algal que en la superficie (FOGG et al., 1973). La scytonemina es un pigmento cianobacterial amaritlo pardusco presente en los estuches extracelulares de algunas especies que florecen en hábitats expuestos a intensa radiación solar y está considerado como un piginento protector de la radiación ultravioleta (DILLON \& CASTENHOLZ 1999). En poblaciones naturales aéreas expuestas de C crustacea de Mendieta y Ligunilia, in scytonemina se presenta predominante en los estuches de las partes más externas o capas superiores de las matas y costras cianobacteriales como ba sido reportado para $C$ c crustacea de Baja California Sur, México (GARCIA-PICHEI \& CASTENHOLZ 1991). Según ABELIOVICH \& SHILO (1972), la protección del sindrome fotoxidativo prodacido por la luz brillante (blanqueado de pigmentos, daño en fotosintesis y muerte celalar) por pigmentos protectores debe ser de valor especial para las especies que enfrentan largos periodos de exposición es decir de especies sujetas a períodos de desecación intermitentes como se presenta en el supramareal. KIRKBY \& WHITTON (1976) también reportaron el desarrollo de pigmentación pardusca en el estuche como una respuesta $\mathrm{a}$ la deficiencia de nutrientes (fierro, magnesio). La pigmentación del estuche exhibida por poblacjones naturales de $C$. crustacea fue perdida en condiciones de cultivo en el laboratorio. Pot consiguiente, ta plasticidad fenotipica de ésta especie en respuesta a cambios en condiciones ambientales fue evidenie coma se ha reportado para otras algas por PEARSON \& KINGSBURY (1966).

$$
\text { Las cianobacterias son }
$$

frecuentemente aturidantes en la zona supramareal de las orillas del mar porque ellas compiten satisfactoriamente con coros 
organismos fotosintéticos en ambientes estresados por limitaciones en el abastecimiento de agua, Las matas laminares cohesivas de $C$. crustacea en Mendieta son perennes, algunas veces aéreas, expuestas en hábitats donde ocasionalmente experimentan desccación. En condiciones adversas el talo de $C$. crustacea empieza a secarse, aunque su estache grueso y mucilaginoso ayuda a reducir la pérdida de agua como en otras especies algales (BONEY 1981). Sin embargo, los talos jueden llegar a desprenderse de la zona supramareal en verano como resultado de la desecación y efecto del viento. El estrés por la desecación es reducido notablemente con el incremento de la marea y la salpicadura producida por rompimiento de las olas modera el efecto adverso de emersión como la baja velocidad metabslica de las algas secas que puede incrementarse dentro de unas pocas hotas cuando los talos se vuelyen a humedecer (FOGG et al., 1973).

La dominancia de una especie en una comunidad algal ha sido atribuido a deficiencia de nutrientes que puede conducir a una competición exclusiva entre las especies algales como fue demostrado por LANGE (1974) cuando Phornidium foweolaram causó la disminución de concentración de fierro y de trazas de metales en el medio de cultivo a niveles muy bajos para el normal crecimiento de otras especies cianobacteriales como Nostac muscorum y Microcystis deruginosa, La deficiencia de nutrientes (cultivos viejos) causada por Oscillatoria $s p$. (Chala) hasta niveles insuficientes para mantener su crecimiento sostenido condujo a su exclusión y a la colonización de sus matas muertas (sustrato) por $C$. cnustacea (sucesión cianobacterial). Además, la estación de crecimiento de cultivos de Oscillatoria sp. fue restringida soblo a unos pocos meses del an̂o (noviembre-marzo), La fuente de nitratos parecen ser el factor principal limitante del crecimiento de algas (MOORE 1974). El aspecto clave del cultivo de Calothrix está en el crecimiento bajo limritación de fosfatos: a niveles no mayores de 1 mg/l (B.A. WHITTON comunicación personal). Este hecho puede explicar el crecimiento sucesional de $C$ crustacea bajo limitacion de nutrientes luego del florecimiento de Oscillatoria sp.. En forma similar, la disminución $y$ posterior exclusión de C. scopuloram coexistiendo inicialmente en cultivos con C. crustacea aparentemente fueron causadas por un abastecimiento insuficiente de ciertos nutrientes, tango de tolerancia a la temperatura ó aleloquímicos.

Aunque el cultivo de algas epilíticas de comunidades complejas en medio definido bajo condiciones controladas de laboratono representa una gran simplificación de las condiciones naturales. la interacción de especies puede conducir a una mejor comprensión de la dinámica poblacional en las orillas rocosas marinas

\section{REFERENCIAS BIBLIOGRAFICAS}

L. Abeliovich, A. y M. Shilo,1972. Photooxydative death in blue green algae: Bacteriol. 111: 682-689.

2. Allsopp, A, 1968. Germination of hormocysts of Scyonema javanicum and the function of blue green algal heterocysts. Nature. London. 220: 810.

3. Boney, A D. 1981. Mucilage: the ubiquitous algal attribute. $\mathrm{Br}$. phycol. J. 16: 115-132,

4. Darley, J., 1968. Contribution a l'étude systématique et biologique des Rivulariacées marines. Boxaniste. I - VI. scrie LI: $141-210$

5. Dillon, J.G. y R. W.Castenholz. 1999. Scytonemin, a cyanobacterial sheath pigment, protects against uve radiation: implications for carly photosyntheric life. J. Phycol. 35, (4):673-681.

6. Dring, M.J., 1982. The Biology of marise plants. E. Amold Lid. p.199.

7. Fogg, G.E.y W.D. Stewart, P. Fay y A.E. Walsby, 1973. The blue green algae. Academic Press Inc. London. p. 298-310.

8. Gallon_J.R. y A.E.Chaplin, 1988. Nitrogen fixation. In L.JRogers \& J.R.Gallon (eds.). Biochemistry of the Algae and Cyanobacteria. Clarendon Press. Oxford, p. 147-173.

9. Garcia-Pichel, F. y R.W. Castenholz, 1991. Characterization and biological implications of scytonemin, a 
cyanobacterial wheath pigment. $J$. Prycol. 27 : 395-409.

10. Golubic, S., 1976. Organism that build stromatolites. In M.R. Walser (ed.). Stromatolites, Elsevier Scientific. Amsterdam. 113-126.

11. Guillard, R.I., 1975. Culture of phyroplankton for fecding marine invertebrates. In W.L. Smith \& M.H. Chanley (eds.), Cuhure of marinc invertebrates animals. Plenum Press. New York. 29-60.

12. Kirkby, S.M. y B.A. Whitton, 1976. Uses of coded data in study of Calothrix and Rivelaria. Br. Phycol. J.Ilt 407. 416.

13. Lange, W., 1974 Competitive exclusion among three plaskionic blue. green algal species. 3. Phycol. 10 (4): $411-414$.

14. Little, C. y J.A. Kitching. 1996. The Biology of Rocky Shores. Oxford University Press. U.K. p.150.

15. Lubchenco, J., 1980. Algal zonstion in the New England rocky intertidal community: an experimental analysis. Ecology, 6 (2): 333-344.

36. Mahasneh, I.A., S.L. Grainger y B.A. Whitton, 1990. Influence of salinity on hair formation anad phosphatase activies of the blue green alga (cyanobocterium) Colothrix vigueri D253. Br. Phycol. J. 25: $24-32$

17. Moore, J.W. 1974, Benthic algae of Southern Baffin Island. III Epilithic and cpilithic communities. J. Phycol. $10(4)$ : 456-462.

18. Pearson, J.E. y J.M. Kingsbury, 1966. Culturally induced variation in four morphologically diverse blue green algae. Am. J. Bot. 53:192-200.

19. Rippka, R., J. Deruelles, J.B. Waterbury, M. Herdman y R. Y. Stainer, 1979. Generic assignments, strain histories and properties of pure cultures of cyanobacteria. J.Gen. Microbiology,-111: 1-61.

20. Sinclair, C. y B.A. Whitton, 1977. Influence of nutrient deficiency on hair formation in the Rivulariaceac. Br. Phycol. J. 12:297-313.

21. Stephenson, T.A. y A. Stephenson, 1949. The universal features of zomation between tidemarks on rocky coasts. $J$. Ecol. 37: 289-305.

22. Stewart, W.D.P., 1965. Nitrogen tumover in marine and brackist habitats. 1. Nitrogen fixation. Am. Bol. 20: 229 239.

23. Vincent, W.F. R.W. Castenholz, M.T. Downes y C. Howard-Williams, 1993. Antartic cyanobacteriat: light, nutrients and photosynthesis in the microbial mat environment. J. Phycol. 29: 745-755.

24. Whitton, B.A. S.M. Kirbky, A. Peat y C. Sinclair, 1973. Exvitonmental effects on the morphology of Rivulariaceac trichomes. In Abstracts of Symposium on Prokaryotic photosynthetic organismx. Freiburge. Br. Germany, 1923.

25. Whitton, B.A., 1992. Diversity, ecology and taxonomy of the Cyanobacteria. In N.H. Mann \& N.G. Cart (eds.). Pholosynthetic Prokaryotes. Plenum Press, New York. 1-51. 